\title{
Afrontamiento y etnia: Estrategias en niños y niñas aymara
}

\section{Coping and ethnicity: Strategies in Aymara boys and girls}

\author{
Alejandra Caqueo-Urízar \\ Departamento de Filosofía y Psicología, Universidad de Tarapacá \\ Department of Social and Behavioral Sciences, Harvard School of Public Health \\ Alfonso Urzúa \\ Escuela de Psicología, Universidad Católica del Norte. \\ Rodrigo Ferrer \\ Departamento de Filosofía y Psicología, Universidad de Tarapacá \\ Noemí Pereda \\ Departamento de Personalidad, Evaluación y Tratamiento Psicológico, Universidad de Barcelona, España \\ Catalina Villena \\ Departamento de Filosofía y Psicología, Universidad de Tarapacá \\ Matías Irarrázaval \\ Departamento de Psiquiatría Norte, Hospital Clínico Universidad de Chile
}

Rec (22 de Julio de 2013) Acept (28 de Abril de 2014)

\begin{abstract}
Resumen
Menores aymara han experimentado un proceso migratorio importante durante las últimas décadas, sin embargo, se han desarrollado escasos estudios en población infantil perteneciente a esta etnia. El objetivo de esta investigación es evaluar y comparar las estrategias de afrontamiento utilizado por niños y niñas Aymara y no Aymara. La muestra estuvo compuesta por 685 niños/as entre 9 y 15 años de escuelas municipales y colegios particular subvencionados de la ciudad de Arica. Los resultados muestran la inexistencia de diferencias poblacionales entre niños/as aymara y no aymara en relación a los problemas experimentados ( $\mathrm{p} \chi 2>.05)$, el uso de estrategias de afrontamiento utilizadas ( $\mathrm{pt}>.05)$ y la eficacia de estas últimas ( $\mathrm{pt}>$ .05 ). Se concluye que los niños/as pertenecientes a la etnia estarían logrando adecuados mecanismos de afrontamiento en contextos urbanos.

Palabras clave:Afrontamiento, etnia, aymara, estrés por aculturación.
\end{abstract}

\begin{abstract}
Aymara Childrens have experienced significant migration process over the past decades, however, few studies have been developed in children belonging to this ethnic group. In this reserch, the objective is to evaluate and compare the coping strategies used by Aymara and not Aymara Children. The sample consisted of 685 boys / girls aged 9 and 15 years of Municipal Schools and Private Schools Funded by the city of Arica.The results show the absence of differences population between Aymara and not Aymara boys and girls, in relation to problems experienced ( $\mathrm{x} \chi 2>.05)$, the use of coping strategies used(pt> .05) and the effectiveness of the latter ( $\mathrm{pt}>.05$ ). We can conclude that children, as belonging to the ethnic group, are achieving adequate coping mechanisms in urban contexts.

Keywords: Coping, ethnicity, Aymara, acculturation stress.
\end{abstract}

Alejandra Caqueo-Urízar, Departamento de Filosofía y Psicología, Universidad de Tarapacá. Avenida 18 de Septiembre \#2222, Arica, Chile. Teléfono: 56-58-2205622, Fax: 56-58-2205818, Email: acaqueo@uta.cl

Agradecimientos: Esta investigación ha sido financiada por el Fondo Nacional de Desarrollo Científico y Tecnológico del Gobierno de Chile, FONDECYT, Proyecto $n^{\circ} 1110472$. 


\section{Introducción}

En Chile conviven una decena de comunidades culturales distintas, a pesar de esto último, muchos de los estudios que se publican en el país no tienen en cuenta las diferentes realidades y características de estos colectivos, tendiendo a generalizar la información obtenida de la población mayoritaria. Entre estos grupos humanos, la comunidad aymara cuenta con una población aproximada de 48.000 personas (INE, 2008), siendo sólo 2.300 personas las que viven en sectores altiplánicos. La mayoría de la población aymara ha emigrado hacia los puertos más cercanos (Arica e Iquique) $\mathrm{y}$ hacia sectores mineros en busca de mejores oportunidades de trabajo (Grebe, 1986; Van Kessel, 1996; Núñez y Cornejo, 2012; González, 2002). Esto ha llevado a que los aymara se han ido adaptando tímidamente a la forma de vida que predomina en las ciudades chilenas, sobre todo los niños, quienes no dejan de sufrir discriminaciones por su fenotipo andino (Kirberg, 2006).

Las familias aymara han tenido que cambiar sus formas de vida, trayendo como consecuencia la pérdida de patrones culturales legendarios (Zapata, 2007). Esto último ha llevado a la vivencia de una crisis de identidad cultural, en la que un alto número de las personas que se podrían definir como aymara por su descendencia o porque aún practican ciertas costumbres originarias, no se consideran como tales, sino como chilenos (90\% según Mamani, 1998). Este proceso de resocialización al que se ve enfrentada la comunidad involucra características psicológicas, tales como cambios de actitudes, valores, normas, adaptación al medio y afiliación grupal que dan paso al concepto de aculturación (Berry, 2002) y que produciría ambigüedad y confusión al sentir el individuo que pertenece a dos culturas distintas de forma simultánea (Dávila y Guarino, 2001).

La tensión emocional originada por el proceso de aculturación puede generar en el individuo un alto nivel de estrés derivado del contacto con una sociedad nueva y los variados retos que comporta (Fajardo, Patiño y Patiño, 2008). Esto es especialmente relevante en los niños, que deben afrontar múltiples estresores derivados de la migración, para los que no siempre tienen recursos (Kirberg, 2006). De este modo, las estrategias de afrontamiento que el menor utilice son fundamentales para su correcta adaptación (Lazarus, 2000).

Sin embargo, los estudios de afrontamiento realizados con niños son escasos y se han caracterizado por obtener resultados no concluyentes respecto a su uso de las diferentes estrategias. Sieffge-Krenke et al. (2001) encontraron diferencias significativas en la percepción de la intensidad de los eventos estresantes en función de la edad. Los autores señalan que los adolescentes mayores (16-17 años) experimentan los problemas relacionados con la escuela como más estresantes de lo que suelen hacer los más pequeños (12-13 años). Por su parte, Escobar, Trianes, FernándezBaena y Miranda (2010) analizaron la asociación de la variable aceptación sociométrica (entendiendo esto como las relaciones interpersonales con iguales) con diversos índices de inadaptación socioemocional, estrés cotidiano y estilo de afrontamiento en niños de entre 9 y 12 años. Los resultados muestran correlaciones significativas negativas de la variable aceptación sociométrica con los índices de inadaptación socioemocional y el estrés cotidiano; además se encontró una correlación significativa positiva con el afrontamiento en 'relación con los demás'. Es decir, el empleo de estrategias de afrontamiento como buscar apoyo social, buscar apoyo profesional, buscar apoyo espiritual, buscar pertenencia, acción social e invertir en amigos íntimos se asocia a una buena aceptación sociométrica (Vega, Villagrán y Nava, 2012).

Dentro de las investigaciones realizadas con niños y niñas de población migrante, Caqueo y Forns (2004) observaron que los niños inmigrantes en España desarrollaban, a una menor edad, más estrategias de aproximación en su proceso de adaptación, buscando además activamente una solución a sus conflictos, al ser comparados con población española. De forma similar, Reinoso y Forns (2010), analizaron las estrategias de afrontamiento en una población de niños adoptados internacionalmente de 8 y 12 años, encontrando que estos emplean un amplio repertorio, siendo la ilusión, regulación emocional, apoyo social, distracción y resolución de problemas las más utilizadas. Por su parte, Samper et al. (2008) indagaron en la existencia de diferencias entre la agresividad y el afrontamiento en los adolescentes españoles y los procedentes de adopciones desde América Latina, Europa Occidental y países árabes, encontrando diferencias en función del lugar de nacimiento en relación a las estrategias de afrontamiento ante un problema, siendo los españoles capaces de buscar apoyo social, invertir en amigos íntimos, buscar pertenencia y distracción física, lo que no se reflejó en los adolescentes pertenecientes a las otras regiones.

Considerando lo anterior, dados los procesos de migración de poblados rurales, se ha ido creando hasta el día de hoy una identidad propia en la etnia aymara que ha sido configurada por la dificultad y complejidad de este proceso en los diferentes ámbitos de ésta (Gundermann, 2000). De este modo, el objetivo de esta investigación es analizar las estrategias de afrontamiento utilizadas por niños y niñas pertenecientes a la cultura aymara, valorando a su vez las 
posibles diferencias en función del sexo, tal y como se ha obtenido en trabajos previos (Stark, Spirito, Williams y Guevremont, 1989; Spirito, Stark, Grace y Stamoulis, 1991) y contribuyendo, de este modo, a un mayor conocimiento de la realidad a la que deben hacer frente estos menores.

\section{Método}

\section{Participantes}

La muestra está constituida por 685 niños y adolescentes de entre 9 y 15 años $(M=11.8 ; D T=1.4)$, de los cuales un $50.2 \%$ son niños $(n=344)$ y un $49.8 \%$ son niñas $(n=341)$.

Los menores aymara corresponden a un $40.1 \%(n=$ $275)$ y los no aymara a un $59.9 \%(n=410)$, no existiendo diferencias entre las proporciones de sexo $(\chi 2 \mathrm{GL}=1=.37$; $\mathrm{p}=.54)$ y edad promedio según el grupo étnico $(\mathrm{tGL}=684=-$ $1.21 ; \mathrm{p}=.23)$.

\section{Procedimiento}

Se realizó un catastro de Escuelas Municipales y Colegios Particulares Subvencionados, que tuvieran un número relevante de alumnos(as) de etnia aymara según listado de beneficiarios de los años 2010-2011 de la Beca Indígena, otorgada por la Junta Nacional de Auxilio Escolar y Becas del Ministerio de Educación. Una vez seleccionados los establecimientos, se les invitó a formar parte de la investigación y se procedió a tomar contacto con la dirección de cada escuela, quienes manifestaron su consentimiento para participar en el estudio. Posteriormente se entrevistó a los profesores jefes del curso a quienes se les explicó los objetivos del estudio. Una vez obtenida la autorización de estos últimos, se invitó a los padres a participar voluntariamente en el estudio. Esto se llevó a cabo durante la primera reunión de apoderados, quienes, luego de ser informados sobre los objetivos de la investigación, firmaron el consentimiento informado. Del mismo modo y considerando la infancia como sujeto de derecho, se solicitó el asentimiento por parte de los niños, es decir, se respetó también la voluntariedad de participar. La evaluación de los niños se realizó en el colegio en horario de clase.

Los criterios de inclusión para el grupo de niños y niñas aymara fueron los siguientes: (a) que el menor tuviera uno o dos apellidos aymara establecidos en la Ley Indígena y (b) la autoadscripción a la etnia aymara, esto es, el reconocimiento como indígena tanto de los padres como del propio niño o niña.

\section{Instrumentos}

Sistema de Codificación de Problemas Vitales (Forns et al., 2004): instrumento que permite una detallada clasificación de los problemas relatados por los sujetos desde una perspectiva analítica y multidimensional, siendo especialmente útil para estudios de relación entre estresores, afrontamiento y salud mental. Los problemas se dividen en 4 dimensiones: naturaleza del problema, participantes, ámbito de ocurrencia y contenido del problema. En cuanto a la fiabilidad entre observadores, el grado de acuerdo entre estos, calculado mediante el índice Kappa de Cohen se encuentra entre .91 y .99 .

Test de Afrontamiento KIDCOPE (Spirito et al., 1988): cuestionario autoaplicado de 15 ítems, que analiza 10 estrategias de afrontamiento divididas en emocionales o de evitación -como son Distracción, Aislamiento, Regulación emocional, Ilusión, Culpar a otros y Resignación- y de aproximación -Restructuración cognitiva, Autocrítica, Apoyo social y Resolución de problemas. Se utilizó la versión para niños entre 7 y 12 años, en una edición experimental para investigación. En esta prueba, el niño debe describir un problema que le haya sido muy preocupante. Posteriormente, el evaluado debe reportar las estrategias utilizadas y valorar su uso y eficacia. Respecto al uso, el menor debe responder ¿hice esto? [sí o no] y para la eficacia debe responder a la cuestión ¿cómo te ayudó?[0: nada, 1: poco, 2: mucho]. En el estudio original, los autores encontraron una fiabilidad test-retest moderada a alta de .41 a .83 . En relación a la validez convergente, se han encontrado valores de entre .33 y .77 entre ítems específicos del Kidcopey otras escalas de afrontamiento.Este cuestionario ha sido ampliamente utilizado en diferentes culturas y países, incluyendo China (Chengy Chan 2003); Alemania (Rathnery Zangerle, 1996; Goldbeck, Bavingy Kohne, 2000); Sudán y Uganda (Paardekooper, de Jongy Hermanns, 1999); Holanda (Jeney-Gammon et al., 1993); Eslovaquia (Ficková, 1998) y España (Caqueo y Forns, 2004; Reinoso y Forns, 2010) mostrando adecuadas propiedades psicométricas para estos colectivos.

\section{Análisis de datos}

Se realizaron análisis descriptivos e inferenciales para variables categóricas y cuantitativas usando la versión 21 del programa IBM SPSS Statistics. 


\section{Resultados}

\section{Características de los problemas reportados}

Las problemáticas que presentaron los participantes, con las cuales se valoraron las estrategias de afrontamiento utilizadas, fueron categorizadas en cuatro componentes del problema: naturaleza del problema, contenido del problema, participantes en el problema y ámbito de ocurrencia del problema.

La naturaleza del problema fue principalmente de tipo 'personal' ( $51.4 \% ; n=351)$ e 'interpersonal' (30.9\%; $n=211)$, mientras que sólo un $17.7 \%(n=121)$ reportó que el problema era de tipo 'ajeno'. No se observaron diferencias poblacionales entre las proporciones de naturaleza del problema ni por sexo $(\chi 2 \mathrm{GL}=2=3.69 ; \mathrm{p}=.16)$ ni por etnia $(\chi 2 \mathrm{GL}=2=.02 ; \mathrm{p}=.99)$.

Los contenidos más frecuentes fueron: relacionales (27.1\%; $n=185)$, vinculados con el rendimiento escolar (15.4\%; $n=105)$, relacionados con la salud $(13.3 \% ; n=91)$, relacionados con la victimización $(5.9 \% ; n=40)$, relacionados con la separación de los padres $(5.7 \% ; n=39)$ y relacionados con la muerte $(5.4 \% ; n=37)$; otras categorías disponibles presentaron proporciones marginales inferiores al $5 \%$ y un $15.5 \%(n=106)$ reportó que el contenido de su problema era 'otros'. Los contenidos observados son distintos en su proporción $(\chi 2 \mathrm{GL}=12=23.45 ; \mathrm{p}=.02)$ entre niños y niñas, principalmente en el ámbito de problemas relacionales (niños $=21.1 \%$; niñas $=33.1 \%$ ) y aquellos relativos a experiencias de victimización (niños $=7.3 \%$; niñas $=4.4 \%$ ). No se observan diferencias poblacionales en los contenidos por etnia $(\chi 2 \mathrm{GL}=12=15.34 ; \mathrm{p}=.22)$.

Los participantes del problema fueron principalmente los propios menores $(39.1 \% ; n=267)$, su núcleo familiar ( $31.0 \% ; n=212)$, compañeros de colegio o amigos $(16.4 \%$; $n=112)$ y miembros de la familia extensa $(7.6 \% ; n=52)$. Se observan diferencias en la proporción de los participantes por sexo $(\chi 2 \mathrm{GL}=7=21.05 ; \mathrm{p}=.00)$, predominando el propio sujeto en los niños (niños $=43.3 \%$; niñas $=34.9 \%$ ) y el núcleo familiar en las niñas (niños $=24.6 \%$; niñas $=37.5 \%$ ). No se observan diferencias poblacionales por etnia en los participantes del problema $(\chi 2 \mathrm{GL}=7=4.04 ; \mathrm{p}=.78)$.

Finalmente, en lo que respecta a las características del problema, la mayoría de ellos ocurren en el hogar u hogar extenso $(35.3 \% ; n=241)$ y en la escuela $(26.9 \% ; n=184)$, aunque un número importante no especifica o no logra especificar el lugar donde ocurre el problema (33.5\%; $n=229$ ). No se observaron diferencias poblacionales entre las proporciones de naturaleza del problema ni por sexo $(\chi 2 \mathrm{GL}=4=7.73 ; \mathrm{p}=.10)$, ni por etnia $(\chi 2 \mathrm{GL}=4=5.42 ; \mathrm{p}=.25)$.

Uso de las estrategias de afrontamiento:

Para conocer el uso de las estrategias de afrontamiento, se calcularon las frecuencias, con la muestra total ( $n=685)$, de cada una de las estrategias de afrontamiento incluidas en el estudio. Los resultados indicaron que las estrategias más utilizadas por la muestra de niños y niñas fueron la 'Ilusión' (91.1\%; $n=624)$; 'Regulación emocional' (89.6\%; $n=614)$; 'Distracción' (85.1\%; $n=583)$; 'Resolución de problemas' ( $81.8 \% ; n=560)$; 'Apoyo social' ( $76.6 \%$; $n=525)$; 'RSEStructuración cognitiva' $(76.2 \% ; n=522)$ y el 'Aislamiento' (59.6\%; $n=408)$. Las menos utilizadas fueron la 'Autocrítica' (41.2\%; $n=282)$, la 'Resignación' (30.9\%; $n=212)$ y 'Culpar a otros' ( $15.3 \% ; n=105)$.

La cantidad media usada de estrategias de tipo aproximativo $(M=3.65 ; D T=1,09)$ y de estrategias evitativas $(M=2.82 ; D T=.98)$ presenta diferencias moderadas $(d=.62)$ (Cohen, 1988) en la población analizada $(\mathrm{p}=.00)$. Además del mayor uso de las estrategias de aproximación respecto al uso de estrategias de evitación, se encuentra que los evaluados consideran que la eficacia o utilidad promedio de las estrategias de aproximación $(M=1.30 ; D T=.45)$ es mayor, en la población general del estudio ( $\mathrm{tGL}=667=6,67 ; \mathrm{p}=.00$ ), que la que ofrecen las estrategias de evitación $(M=1.18 ; D T=$ .49), aunque la diferencia es mínima ( $d=.24)$ (Cohen, 1988).

Para valorar las diferencias en el uso de estrategias de afrontamiento entre niños $(n=344)$ y niñas $(n=341)$, se realizó una tabla de contingencias contrastando la hipótesis de independencia con el estadístico $\chi^{2}$ de Pearson para cada una de las estrategias evaluadas. Los resultados se presentan en la tabla 1.

A nivel general, se aprecia que las niñas $(M=3.74$; $D T=1.03)$ respecto a los niños $(M=3.56 ; D T=1.13)$ utilizan más estrategias de aproximación ( $\mathrm{tGL}=678,55=-2.18$; $\mathrm{p}=$ $.03)$, aunque el efecto de dicha diferencia es marginal $(d=-$ .17) (Cohen, 1988). En cambio, no se observan diferencias en el uso de estrategias de evitación ( $\mathrm{tGL}=683=.13 ; \mathrm{p}=.89$ ). Se observa que niños y niñas usan las distintas estrategias en la misma proporción, salvo el uso más frecuente de 'RSEStructuración cognitiva' $(\chi 2 \mathrm{GL}=1=20.36 ; \mathrm{p}=.00)$ y 'Apoyo social' $(\chi 2 \mathrm{GL}=1=3.70 ; \mathrm{p}=.05)$ por parte de las niñas.

Respecto a la percepción de eficacia entre niños y niñas, se observa que las niñas $(M=1.34 ; D T=.44)$ valoran como más eficaces ( $\mathrm{tGL}=675=-2.71 ; \mathrm{p}=.01$ ) las estrategias de aproximación en comparación a los niños $(M=1.25 ; D T=.47)$, si bien esta diferencia es marginal $(d=-, 22)$ (Cohen, 1988). En cambio, en la percepción de eficacia de las estrategias 
Tabla 1.Proporción y contraste de independencia de las estrategias de afrontamiento según sexo

\begin{tabular}{lcccc}
\hline Estrategia & \multicolumn{2}{c}{ Proporción de uso } & & \\
& Niños & Niñas & $\chi 2 \mathrm{GL}=1$ & $\mathrm{p}$ \\
& & $\%(\mathrm{n})$ & & \\
\hline Distracción & $84.0 \%(289)$ & $86.2 \%(294)$ & .66 & .42 \\
Aislamiento & $58.7 \%(202)$ & $60.4 \%(206)$ & .20 & .65 \\
RSEStructuración cognitiva & $68.9 \%(237)$ & $83.6 \%(285)$ & 20.36 & .00 \\
Autocrítica & $44.2 \%(152)$ & $38.1 \%(130)$ & 2.60 & .11 \\
Culpar a otros & $16.6 \%(57)$ & $14.1 \%(48)$ & .82 & .37 \\
Resolución de problemas & $80.2 \%(276)$ & $83.3 \%(284)$ & 1.07 & .30 \\
Regulación emocional & $89.5 \%(308)$ & $89.7 \%(306)$ & .01 & .93 \\
Ilusión & $89.2 \%(307)$ & $93.0 \%(317)$ & 2.92 & .09 \\
Apoyo social & $73.5 \%(253)$ & $79.8 \%(272)$ & 3.70 & .05 \\
Resignación & $34.0 \%(117)$ & $27.9 \%(95)$ & 3.03 & .08 \\
\hline
\end{tabular}

Tabla 2.Proporción y contraste de independencia de las estrategias de afrontamiento según pertenencia étnica

\begin{tabular}{lcccc}
\hline Estrategia & \multicolumn{2}{c}{ Proporción de uso } & $\chi 2 \mathrm{GL}=1$ & $\mathrm{p}$ \\
& $\begin{array}{c}\text { Aymaras } \\
\%(\mathrm{n})\end{array}$ & $\begin{array}{c}\text { No Aymaras } \\
\%(\mathrm{n})\end{array}$ & & \\
\hline Distracción & $85.5 \%(235)$ & $84.9 \%(348)$ & .04 & .84 \\
Aislamiento & $59.3 \%(163)$ & $58.8 \%(245)$ & .02 & .90 \\
RSEStructuración cognitiva & $76.0 \%(209)$ & $76.3 \%(313)$ & .01 & .92 \\
Autocrítica & $40.4 \%(111)$ & $41.7 \%(171)$ & .12 & .73 \\
Culpar a otros & $14.9 \%(41)$ & $15.6 \%(64)$ & .06 & .80 \\
Resolución de problemas & $80.7 \%(222)$ & $82.4 \%(338)$ & .32 & .57 \\
Regulación emocional & $90.9 \%(250)$ & $88.8 \%(364)$ & .80 & .37 \\
Ilusión & $92.0 \%(253)$ & $90.5 \%(371)$ & .46 & .50 \\
Apoyo social & $77.8 \%(214)$ & $75.9 \%(311)$ & .36 & .55 \\
Resignación & $32.0 \%(88)$ & $30.2 \%(124)$ & .24 & .66 \\
\hline
\end{tabular}

de evitación, no existen diferencias ( $\mathrm{tGL}=669=-.92 ; \mathrm{p}=.36)$ entre niños $(M=1.16 ; D T=.50)$ y niñas $(M=1.20 ; D T=.49)$.

En el caso de la categoría etnia, para valorar las diferencias en el uso de estrategias de afrontamiento entre aymara $(n=275)$ y no aymara $(n=410)$, se contrastó la hipótesis de independencia para cada una de las estrategias de afrontamiento. Los resultados se presentan en la tabla 2.

No se observan diferencias en función del grupo étnico en el uso promedio de estrategias de aproximación $(\mathrm{tGL}=683=.08$; $\mathrm{p}=.94)$, ni de evitación ( $\mathrm{tGL}=683=.35 ; \mathrm{p}=.73$ ). Ninguna de las estrategias de afrontamiento evaluadas permite rechazar la hipótesis de independencia entre grupos, por lo que debe asumirse que las estrategias utilizadas no tienen relación con el grupo étnico. En lo que respecta a la valoración de la eficacia de las estrategias de afrontamiento usadas, tampoco se observan diferencias en la valoración que hacen aymaras y no aymaras en las estrategias de aproximación $(\mathrm{tGL}=675=-$ $.89 ; \mathrm{p}=.38$ ) y de evitación ( $\mathrm{tGL}=669=-.34 ; \mathrm{p}=.73$ ).

Finalmente, ante la posibilidad que las diferencias o no diferencias detectadas se deban a efectos de interacción entre sexo y etnia, se procedió a valorar la interacción de ambas categorías en el uso y eficiencia de las estrategias de interacción por aproximación y de evitación. Para esto, se realizó un análisis de varianza usando la interacción de las categorías sexo y etnia como variables independientes.

Los resultados indican que, manteniendo la hipótesis de igualdad de varianza para todas las variables dependientes (probabilidad del estadístico de Levene mayor a .05 en todos los casos), no existen efectos de interacción en el uso de estrategias de afrontamiento por aproximación $(\mathrm{FGL}=1=1.44$; 
$\mathrm{p}=.28)$ y evitación $(\mathrm{FGL}=1=.51 ; \mathrm{p}=.50)$. Tampoco se observan efectos de interacción en lo que respecta a la eficacia percibida de las estrategias de aproximación $(\mathrm{FGL}=1=.36$; $\mathrm{p}=.51)$ y evitación $(\mathrm{FGL}=1=.25 ; \mathrm{p}=.63)$.

\section{Discusión}

El presente estudio muestra las estrategias de afrontamiento que niños y niñas aymara presentan ante las múltiples demandas que su nuevo entorno, como minoría étnica a la que pertenecen, les exige. Se trata de uno de los primeros trabajos en describir las características de un colectivo escasamente estudiado.

La masiva migración hacia la costa y el rápido abandono de los poblados rurales precordilleranos ha sido y sigue siendo una de las experiencias más relevantes para la población aymara, debido a que no implica una salida sin retorno, ya que se mantienen los nexos con las comunidades de origen (Gunderman, 2000).

Durante todo el siglo XX la cultura aymara sufrió el poderoso impacto del Estado Chileno en la Región de Arica y Parinacota, principalmente a través del desarrollo de obras públicas, la expansión del sistema escolar y la conscripción militar. En este sentido, la apertura de caminos y otros programas de inversión en infraestructura aceleraron la integración económica y política de las zonas interiores a los centros regionales; a través de la escuela se difundió la cultura nacional y el uso del castellano; el servicio militar incentivó la adhesión a los valores patrios y proporcionó nuevas experiencias migratorias a las nuevas generaciones. De este modo, la intervención del Estado y sus agentes trajo como consecuencia una asimilación cultural (Gundermann, González y Vergara, 2007). Esto último es coherente con los resultados obtenidos en el estudio, observándose que en ninguna de las categorías señaladas por los menores, esto es, naturaleza del problema, contenidos más frecuentes, participantes del problema y características de estos últimos, se observaron diferencias entre niños y niñas aymara y aquellos no pertenecientes a esta etnia. Resultados similares se encontraron en relación a las estrategias de afrontamiento, donde tampoco hubo diferencias entre niños y niñas aymara en cuanto al uso de la estrategia, tanto de aproximación como evitación, ni en la valoración de la eficacia de las estrategias utilizadas, respecto al grupo culturalmente mayoritario.

Estos resultados, no frecen apoyo a la hipótesis inicial de los investigadores y, sin duda, generan discusión.En primer lugar, es posible que el 'estrés por aculturación' podría no estar dándose en estos niños y por ende, tampoco exista una disminución en su capacidad de afrontar, incluso podría estar generando mejoría en varios aspectos de sus vidas (Sam y Berry, 1995). Así, algunos autores describen ciertas 'ventajas interculturales' que caracterizan a los grupos subalternos 'forzados' a procesos históricos de 'aculturación' (De Munter, 2007). Esto es coherente con un estudio realizado con niños inmigrantes en Barcelona donde estos últimos, a menor edad, habían desarrollado más estrategias de aproximación en su proceso de adaptación, buscando además activamente una solución a sus conflictos (Caqueo y Forns, 2004). Se suma a esto último, una investigación realizada por Sobral et al. (2010), quienes analizaron las estrategias utilizadas por un grupo de adolescentes Latinoamericanos en España, encontrando que las más empleadas fueron las de integración y asimilación de la cultura española. Desde una perspectiva antropológica, las dinámicas de la aculturación también pueden resultar en procesos ambiguos pero constructivos de 'creolización'. Esto quiere decir que, en esta muestra de niños aymara y no aymara, se estarían generando zonas de contacto - 'spaces in between' (Bhabha) o 'interfases entre sistemas' (Temple et al., 2003) entre diferentes prácticas o ámbitos culturales (De Munter, 2007). De estas zonas podrían estar surgiendo maneras interesantes de combinar diferentes estrategias de vida que los lleva a desarrollar un adecuado afrontamiento.

Cabe tener en cuenta, como segunda hipótesis, que, efectivamente, si bien estos niños son aymara, han sido trasladados desde el altiplano hace bastante tiempo, por lo que han aprendido a vivir entre dos culturas: la de sus antepasados y la actual urbana. Un estudio realizado con un grupo de inmigrantes Sudamericanos en Chile, reveló que los que logran integrarse a la cultura de destino presentan mejores indicadores en las dimensiones de bienestar psicológico, autonomía, propósito vital y relaciones sociales (Yáñez y Cárdenas, 2010). Por lo tanto los hallazgos parecen apoyar que estos niños y niñas han logrado mecanismos adecuados de adaptación que les han permitido integrarse al contexto urbano. Estos resultados también son consistentes a lo encontrado por Zwirs et al. (2007) con niños inmigrantes en Europa y por Costello et al. (1997) con niños inmigrantes en Estados Unidos.

Una tercera posibilidad es que no existan diferencias en las formas de afrontamiento en estos niños aymara debido a que aún se encuentran en su infancia, pero que puede que surjan diferencias conforme los niños vayan creciendo y deban afrontar una mayor discriminación (Zwirs, et al., 2007). El rango de edad de la muestra, y las características trasversales del estudio, impiden poder analizar esta opción en 
profundidad, siendo necesario incorporar aspectos evolutivos en futuras evaluaciones (Urzúa y Caqueo-Urízar, 2012).

Respecto a los resultados obtenidos en referencia al sexo, las niñas reportaron más problemas relacionales y con la familia, en cambio los niños señalaron más problemas personales y de victimización. Estos resultados se encuentran en línea con otros trabajos internacionales que han utilizado una metodología similar (Forns, Amador, Kirchner, Martorell, Zanini y Muro, 2004; Pereda, Forns, Kirchner y Muñoz, 2009). Además, las niñas utilizan más estrategias de aproximación que los niños, lo que también es coherente con estudios previos (Pereda et al., 2009).

Las diferencias respecto al sexo en el uso de las estrategias de afrontamiento ha sido constatada en múltiples trabajos (para una revisión véase Tamres, Janicki y Helgeson, 2002). Sin embargo, su eficacia ha sido poco estudiada (Donaldson, Prinstein, Danovsky y Spirito, 2000). En este sentido, los resultados del presente estudio apuntan a que existen diferencias significativas en la eficacia que niños y niñas atribuyen a las estrategias de afrontamiento utilizadas. Sin embargo, no se han encontrado diferencias significativas en función del sexo respecto a la eficacia del afrontamiento en trabajos previos (Pereda et al., 2009), lo que apunta a la necesidad de nuevos estudios que aporten más información a este respecto.

Dentro de las principales limitaciones del presente estudio cabe mencionar que sólo se evaluó a niños y niñas aymara de Chile, por lo que los resultados no pueden ser generalizables a toda la población aymara infantil, sugiriendo además la necesidad incorporar en futuros estudios a niños y niñas de otras etnias, tanto dentro del país como mapuches, rapa nuis o quechuas o fuera de éste. Si bien la muestra analizada no puede considerarse representativa del colectivo de niños y niñas aymara, el uso de una muestra probabilística y el tamaño de la misma, apoyan la extracción de conclusiones a partir del colectivo analizado. En segundo lugar, es probable que existan algunas dificultades cuando se utilizan conceptos psicológicos e instrumentos de medición pertenecientes a otras culturas (Bracken, et al., 1995; Derluyn, et al., 2008). A este respecto, cabe tener en cuenta, no obstante, que la comparativa de los resultados con los obtenidos por trabajos de otros países con la misma metodología, parece apuntar a la validez de estos instrumentos en el contexto chileno. Finalmente, el estudio presenta un diseño transversal, en el cual los datos se obtuvieron en un momento determinado, sin considerar aquellos factores que pudieran haber afectado a la evaluación, por lo que sería importante realizar estudios longitudinales que permitan evaluar la consistencia de los hallazgos obtenidos a lo largo del tiempo (Urzúa, 2013).

\section{Conclusiones}

La pertenencia a la etnia aymara en niños y niñas residentes en la ciudad de Arica no genera diferencias en las estrategias de afrontamiento utilizadas, sin embargo, cuando se considera la muestra total, se observan diferencias por sexo en cuanto al uso de estas estrategias. Es probable que los niños y niñas aymara estén desarrollando mecanismos adecuados de adaptación, por lo que más allá de un 'estrés por aculturación', se debiera pensar en 'procesos de transculturación’ y de manera más general, de nuevas dinámicas interculturales que pueden tener efectos positivos en los niños y niñas.

\section{Referencias}

Berry, J.W. (2002). Conceptual approaches to acculturation. In G. Marín, P. Balls-Organista, and K. Chung (Eds), Acculturation (pp. 17-37) Washington, DC: APA Books.

Caqueo, A., y Forns, M. (2004). Estrategias de afrontamiento en niños inmigrantes escolarizados en Barcelona. En E. Doménech, J. Obiols, M. Jané y S. Subirá (Eds.), Actualizaciones en Psicopatología clínica (pp.49-61). Barcelona: Universitat Autónoma de Barcelona.

Bracken, P., Giller, J., y Summerfield, D. (1995). Psychological responses to war and atrocity: The limitations of the current concepts. Social Sciencey Medicine, 40, 1073-1082.

Cheng, S., y Chan, A. (2003). Factorial structure of the Kidcope in Hong Kong adolescents. The Journal of Genetic Psychology, 164, 261-266.

Cohen, J. (1988). Statistical power analysis for the behavioral sciences $\left(2^{a}\right.$ ed.) New York: Academic Press.

Costello, E. J., Farmer, E. M., Angold, A., Burns, B. J., y Erkanli, A. (1997). Psychiatric disorders among American Indian and white youth in Appalachia: the Great Smoky mountains study. Amer J Public Health 87, 827-832.

Dávila, B., y Guarino, L. (2001). Fuentes de estrés y estrategias de afrontamiento en escolares Venezolanos. Revista Interamericana de Psicología, 31, 97-112.

De Munter, K. (2007). Nayra.Ojos al Sur del Presente. Aproximaciones antropológicas a la interculturalidad contemporánea. Latinas Editores - CEPA, Oruro.

Derluyn, I., Broekaert, E., y Schuyten, G. (2008). Emotional and behavioural problems inmigrant adolescents in Belgium European Child and Adolescent Psychiatry, 17, 54-62.

Donaldson, D., Prinstein, M. J., Danovsky, M., y Spirito, A. (2000). Patterns of children's coping with life stress: implications for clinicians American Journal of Orthopsychiatry, 70, 351-359.

Escobar, M. Trianes, M., Fernández-Baena, F., y Miranda, J. (2010). Relaciones entre aceptación sociométrica escolar e inadaptación socioemocional, estrés cotidiano y afrontamiento. Revista Latinoamericana de Psicología, 42, 469-479.

Fajardo, M., Patiño, M. I., y Patiño, C. (2008). Estudios actuales sobre aculturación y salud mental en inmigrantes: Revisión y perspectivas. Revista Iberoamericana de Psicología, Ciencia y Tecnología, 1, 39-50.

Ficková, E. (1998). Coping scales for children and adolescents: psychometric relationships. Studia Psychologica, 40, 291-296.

Forns, M., Amador, J. A., Kirchner, T., Martorell, B., Zanini, D., y Muro, P. (2004) Sistema de codificación y análisis diferencial de los problemas de los adolescentes [Codification system and differential analysis of problems related by adolescents]. Psicothema, 16, 647-654. 
Goldbeck, L., Baving, A., y Kohne, E. (2000) PsychosozialeAspekte der b-Thalassämie: Krankheitserleben, CopingundTherapiemitarbeit. (Psychosocial aspects of beta-thalassemia: distress, coping and adherence). Klinische Pädiatrie, 212, 254-259.

González, H. (2002). Los Aymaras contemporáneos. En A.M. Carrasco y B. Cofré (Eds.), Conozcamos juntos la Historia y Cultura de Nuestra Región. (pp. 39-45) Edición TEA-EXPLORA-UTA, Arica.

Grebe, M. E. (1986). Migración, identidad y cultura Aymara: Puntos de vista del actor. Revista Chungara, 16-17, 205-223.

Gundermann, H. (2000). Las organizaciones étnicas y el discurso de la identidad en el norte de Chile, 1980-2000. Estudios Atacameños, 19, 75-91.

Gundermann, H., González, H., y Vergara, J. (2007). Vigencia y desplazamiento de la lengua aymara en Chile. Estudios Filológicos 42, 123-140.

INE (Instituto Nacional de Estadísticas (2008). Población y Sociedad: Aspectos demográficos. Santiago, Chile: Instituto Nacional de Estadísticas.

Jeney-Gammon, P., Daugherty, T. K., Finch, A. J., Belter, R.W., y Foster, K. Y. (1993). Children's coping styles and report of depressive symptoms following a natural disaster. Journal of Genetic Psychology, 154, 259-267.

Kirberg, A. (2006). La salud del niño Aymará. Revista Chilena de Pediatría, 77, 608-609.

Lazarus, R. (2000). Estrés y Emoción. Manejo e implicaciones en nuestra salud. Bilbao. Biblioteca de Psicología Desclée de Brouwer.

Mamani, G. (1998). Educación y Lengua Aymara en el Norte de Chile. Recuperado desde http://www.puebloindio.org/ONU info/info98/ GTP198_aymarka.htm

Núñez, R. E. y Cornejo, C. (2012). Facing the Sunrise: Cultural Worldview Underlying Intrinsic-Based Encoding of Absolute Frames of Reference in Aymara. Cognitive Science, 36: 965-991.

Paardekooper, B., de Jong, J. T. V. M., y Hermanns, J. M. A. (1999). The psychological impact of war and the refugee situation on South Sudanese children in refugee camps in Northern Uganda: an exploratory study. Journal of Child Psychology and Psychiatry, 40, 529-536.

Pereda, N., Forns, M., Kirchner, T., y Muñoz, D. (2009). Classification and analysis of children's problems and coping strategies with the Kidcope. Child: Care, Health and Development, 35, 841-850.

Rathner, G., y Zangerle, M. (1996). Copingstrategienbei Kinder und Jugendlichenmit Diabetes mellitus: Die deutschsprachige Version des Kidcope. (Coping strategies of children and adolescents with diabetes mellitus: the German language version of Kidcope). Zeitschriftfür Klinische Psychologie Psychiatrie und Psychotherapie, 44, 49-74.

Reinoso, M., y Forns, M. (2010). Estrés, estrategias de afrontamiento y puntos Fuertes y dificultades: Un estudio sobre menores adoptados internacionalmente en Cataluña. Revistas de Psicología. Ciencies de I'Educació i de I'Esport, 27, 141-150.

Sam, D. L., y Berry, J. W. (1995). Acculturative stress amongyoung immigrants in Norway. Scandinavian Journal of Psychology, 36, 10-21.
Samper, P., Tur, A., Mestre, V., y Cortés M. T. (2008). Agresividad y afrontamiento en la adolescencia. Una perspectiva intercultural. International Journal of Psychology and Psychological Therapy, 8, 431-440.

Sieffge-Krenke, I., Weidermann, S., Fentner, S., Aegenheister, N., y Poeblau, M. (2001). Coping with school-related stress and family stress in healthy and clinically referred adolescents. European Psychologist, $6,123-132$.

Sobral, J., Gómez-Fraguela, J.A., Luengo, A., Romero, E., y Villar, P. (2010). Adolescentes latinoamericanos, aculturación y conducta antisocial. Psicothema, 22, 410-415.

Spirito, A., Stark, L. J., y Williams, C. (1988). Development of a brief coping checklist for use with pediatric populations. Journal of Pediatric Psychology, 13, 555-574.

Spirito, A., Stark, L. J., Grace, N., y Stamoulis, D. (1991). Common problems and coping strategies reported in childhood and early adolescence. Journal of Youth and Adolescence, 20, 531-544.

Stark, L. J., Spirito, A., Williams, C. A., y Guevremont, D. C. (1989). Common problems and coping strategies I: findings with normal adolescents. Journal of Abnormal Child Psychology, 17, 203-212.

Tamres, L. K., Janicki, D., y Helgeson, V. S. (2002) Sex differences in coping behavior: a meta-analytic review and an examination of relative coping. Personality and Social Psychology Review, 6, 2-30.

Temple, D., Layme, F., Michaux, J., González, M., y Blanco, E. (2003). Las estructuras elementales de la reciprocidad. TARI, Plural Editores, La Paz.

Urzúa, A., y Caqueo-Urízar, A. (2012). “Calidad de Vida: Una revisión teórica del concepto". Terapia Psicológica, 30, 61-71.

Urzúa, A., Julio, C., Páez, D., Sanhueza, J., y Caqueo-Urízar, A. (2013). “¿Existen diferencias en la evaluación de la calidad de vida cuando los menores valoran la importancia de lo que se les pregunta?". Archivos Argentinos de Pediatría, 111, 98-104.

Van Kessel, J. (1996). Los Aymaras contemporáneos de Chile. In J. Hidalgo, F. Schiappacasse, H. Niemeyer, C. Aldunate y P. Mege (Eds.), Etnografia: Sociedades indigenas contemporáneas y su ideología (pp. 47-67). Santiago, Chile: Editorial Andrés Bello.

Vega, C., Villagrán, M. y Nava, C. (2012). Estrategias de afrontamiento, estrés y depresión infantil. Revista PsicologiaCientifica.com, 14 (21). Disponible en: http://www.psicologiacientifica.com/estrategias-afrontamiento-estres-depresion-infantil

Yañez, S., y Cárdenas, M. (2010). Estrategias de aculturación, indicadores de salud mental y bienestar psicológico en un grupo de inmigrantes sudamericanos en Chile. Salud y Sociedad, 1, 51-70.

Zapata, C. (2007). Memoria e historia. El proyecto de una identidad colectiva entre los Aymará de Chile. Chungara. Revista de Antropología Chilena, 39, 171-183.

Zwirs, B., Burger, H., Schulpen, T., Wiznitzer, M., Fedder, H., y Buitelaar, J. (2007). Prevalence of Psychiatric Disorders Among Children of Different Ethnic Origin. Journal Abnorm Child Psychol, 35, 556-566. 